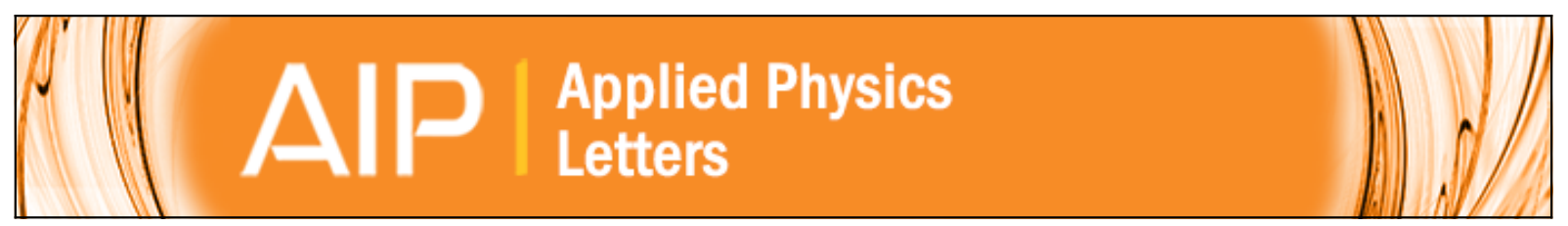

\title{
Strain release management in SiGe/Si films by substrate patterning
}

V. Mondiali, M. Bollani, D. Chrastina, R. Rubert, G. Chahine, M. I. Richard, S. Cecchi, L. Gagliano, E. Bonera, T.

Schülli, and L. Miglio

Citation: Applied Physics Letters 105, 242103 (2014); doi: 10.1063/1.4904455

View online: http://dx.doi.org/10.1063/1.4904455

View Table of Contents: http://scitation.aip.org/content/aip/journal/apl/105/24?ver=pdfcov

Published by the AIP Publishing

\section{Articles you may be interested in}

Metastability and relaxation in tensile $\mathrm{SiGe}$ on $\mathrm{Ge}(001)$ virtual substrates

J. Appl. Phys. 116, 113507 (2014); 10.1063/1.4896076

Strain relaxation of metastable SiGe/Si: Investigation with two complementary X-ray techniques

J. Appl. Phys. 111, 063507 (2012); 10.1063/1.3694037

SiGe relaxation on silicon-on-insulator substrates: An experimental and modeling study

J. Appl. Phys. 94, 7892 (2003); 10.1063/1.1628406

SiGe-free strained Si on insulator by wafer bonding and layer transfer

Appl. Phys. Lett. 82, 4256 (2003); 10.1063/1.1581371

Relaxation of strained Si layers grown on SiGe buffers

J. Vac. Sci. Technol. B 17, 1424 (1999); 10.1116/1.590771

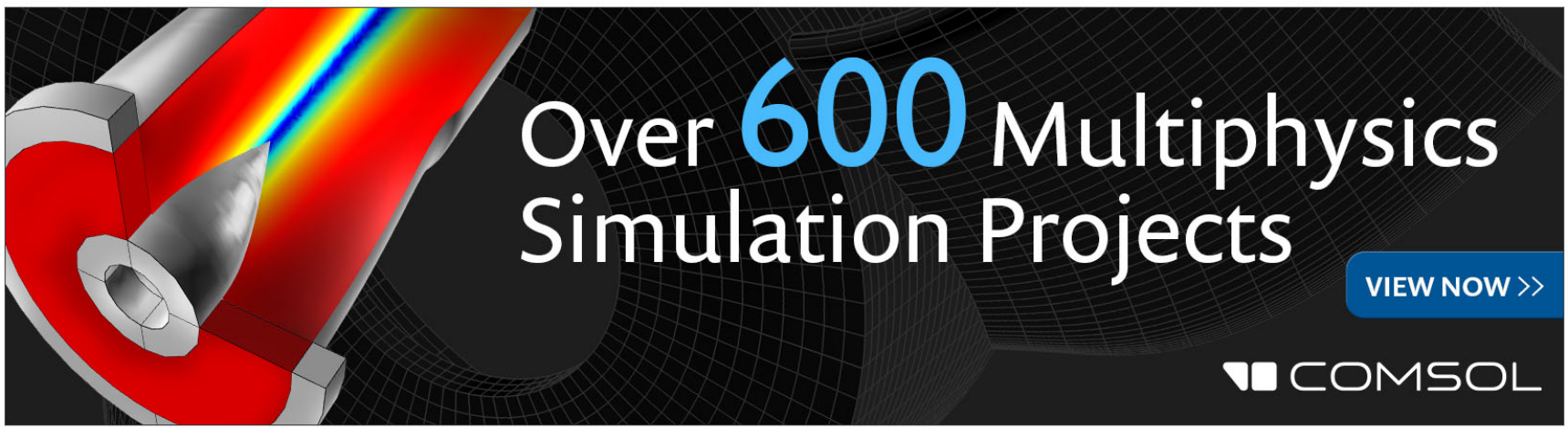




\title{
Strain release management in SiGe/Si films by substrate patterning
}

\author{
V. Mondiali, ${ }^{1}$ M. Bollani, ${ }^{2, a)}$ D. Chrastina, ${ }^{1}$ R. Rubert, ${ }^{1}$ G. Chahine,${ }^{3}$ M. I. Richard,${ }^{3,4}$ \\ S. Cecchi, ${ }^{1}$ L. Gagliano, ${ }^{5}$ E. Bonera,${ }^{5}$ T. Schülli, ${ }^{3}$ and L. Miglio ${ }^{5}$ \\ ${ }^{1}$ L-NESS, Dipartimento di Fisica, Politecnico di Milano, via Anzani 42, 22100 Como, Italy \\ ${ }^{2}$ IFN-CNR, L-NESS, via Anzani 42, 22100 Como, Italy \\ ${ }^{3}$ ID01/ESRF, BP 220, F-38043 Grenoble Cedex, France \\ ${ }^{4}$ Aix-Marseille Universite, CNRS, IM2NP UMR 7334, Campus de Saint Jerome, \\ F-13397 Marseille Cedex, France \\ ${ }^{5}$ L-NESS and Department of Material Science, University of Milano-Bicocca, via Cozzi 55, \\ I-20125 Milano, Italy
}

(Received 14 November 2014; accepted 2 December 2014; published online 15 December 2014)

\begin{abstract}
The nucleation and the evolution of dislocations in $\mathrm{SiGe/Si}(001)$ films can be controlled and confined along stripes aligned along pits carved in the substrate, leaving micrometric coherent areas free of dislocations. In this work, we have addressed the stability of such metastable areas versus, film thickness, different $\mathrm{Ge}$ contents $\left(\mathrm{x}_{\mathrm{Ge}}=10 \%-30 \%\right)$ and larger pit-pattern periods, revealing the flexibility and effectiveness of this method even for coherent areas of about $64 \mu \mathrm{m}^{2}$. The thermal stability of such configuration has been finally verified by post-growth annealing treatment, in order to simulate device processing. Finally, $\mu$ Raman spectroscopy and X-ray nanodiffraction have been used to characterize the periodic strain variations across the pattern. (C) 2014 AIP Publishing LLC. [http://dx.doi.org/10.1063/1.4904455]
\end{abstract}

The introduction of $\mathrm{SiGe} / \mathrm{Si}$ structures into $\mathrm{Si}$-based information and communications technology industries ${ }^{1}$ offered the opportunity to overcome the power crisis, but it has been limited to very thin layers, for instance, within heterojunction bipolar transistors ${ }^{2,3}$ or as local stressors for strained Si channel transistors. ${ }^{4}$ The possibility of using partially relaxed thicker SiGe epilayers for strained Ge technology is hindered by the random nucleation of dislocations and threading arms bunches of them, due to the unavoidable plastic relaxation of the material. ${ }^{5}$ Threading arms impact negatively on device performance, ${ }^{6}$ and the device yield becomes unpredictable because of the random nature of the nucleation process. Hence, any methods of controlling and confining the dislocations to selected areas are highly welcome. It has been shown that $\{111\}$-faceted pits lead to the trapping of dislocations during $\mathrm{SiGe}$ epitaxial growth at the onset of plastic relaxation. ${ }^{7,8}$ In these works, it was shown that the patterning strongly influences the nucleation and the propagation of dislocations, preferentially segregating the misfit segments along pit rows and the threading arms inside the pits. However, it is still necessary to optimize the process to obtain useful coherent areas of strained SiGe between the dislocated stripes, by exploring the influence of the pit spacing, in relation to the Ge content and the average degree of relaxation. We also show how the SiGe film grown on a suitable pit pattern is kinetically stable with further annealing treatments, which is relevant to device applications such as novel vertical devices ${ }^{9}$ and optoelectronic applications. ${ }^{10}$ Since this metastable configuration depends also on the growth method, different growth conditions have been considered. Misfit dislocation accumulation in stripes, separated by coherent areas, are revealed at the film surface by atomic force microscopy (AFM), in place of the usual

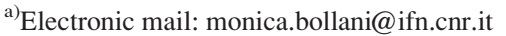

uniform cross hatch, but the confirmation comes from suitable nano X-ray diffraction maps. In addition, $\mu$ Raman spectroscopy maps are reported, indicating the sharp periodicity of strain release in agreement to modest average relaxation measured by X-ray diffraction (XRD). ${ }^{8,11}$ The $\mathrm{SiN}_{x} / \mathrm{Si}$ (001) substrate is patterned with arrays of $\{111\}$-faceted pits aligned along the $\langle 110\rangle$ directions by electron beam lithography and reactive ion etching. Pits with the shape of inverted $\{111\}$-faceted pyramids were then formed using a selective wet-etching, as described in Refs. 8 and 12. The typical width of the pits after etching is about $750 \mathrm{~nm}$ with a corresponding depth of $600 \mathrm{~nm}$, while patterns are designed with pitches between 2 and $10 \mu \mathrm{m}$. Then, a $30 \mathrm{~nm}$ Si buffer and a $190 \mathrm{~nm} \mathrm{Si}_{1-x} \mathrm{Ge}_{x}$ alloy layer (with a Ge content of 15\%-30\%) were deposited by low-energy plasma-enhanced chemical vapor deposition (LEPECVD) at temperatures in a range of $550-700{ }^{\circ} \mathrm{C}$ and with a growth rate of about $0.4 \mathrm{~nm} / \mathrm{s}$. In this way, a conformal $\mathrm{SiGe}$ film, thicker than the equilibrium critical thickness ${ }^{5,13}$ for plastic relaxation onset, is achieved.

First, we evaluated the effect of the Ge content keeping constant the thickness of the layer, the growth temperature, and the growth rate. Samples with $190 \mathrm{~nm}$ thick $\mathrm{Si}_{1-\mathrm{x}} \mathrm{Ge}_{\mathrm{x}}$ $(x=12 \%-30 \%)$ were grown on a $30 \mathrm{~nm} \mathrm{Si}$ buffer layer at growth temperature of $650{ }^{\circ} \mathrm{C}$ and a nominal growth rate of $0.38 \mathrm{~nm} / \mathrm{s}$. Although at Ge contents higher than $20 \%-25 \%$ the nucleation of quantum dots is usually expected, as one elastic path to strain relaxation, all the layers are here expected to partially relax by $60^{\circ}$ dislocations, since at minor lattice misfits the driving stress to complete plastic relaxation is less effective. ${ }^{14}$ This is possible because the LEPECVD technique allows the effect of the growth temperature and of the growth rate to be decoupled, offering the possibility to use sufficiently low temperatures and high rates. The final result is always a continuous film with delayed plastic 


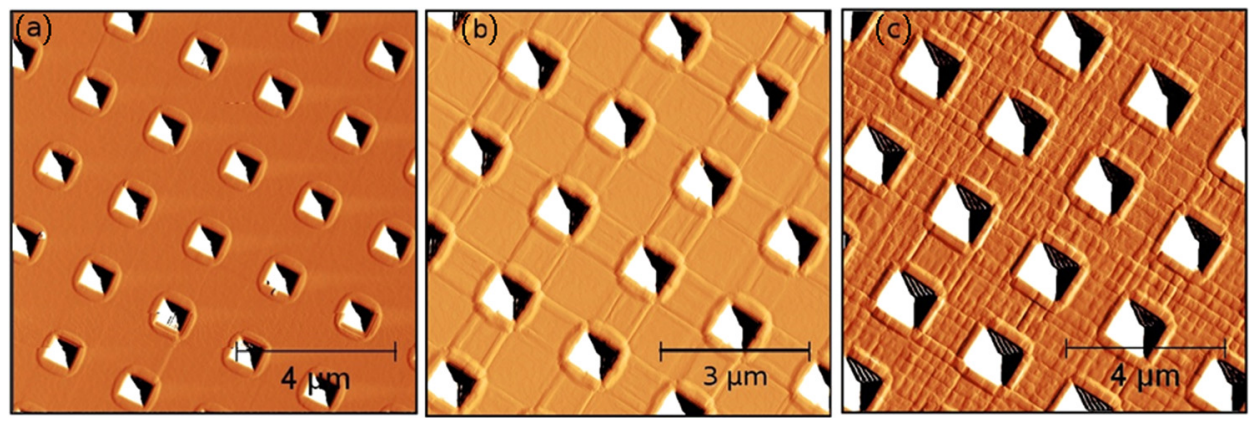

FIG. 1. AFM tapping-amplitude images, following the deposition of $190 \mathrm{~nm}$ of SiGe on pit-patterned substrates. (a) Ge content of about $12.6 \%$, $\beta$ of about $29.1 \%$, and a pitch of about $2 \mu \mathrm{m}$ : a few surface step lines joining adjacent pit corners. (b) Ge content of about $20.4 \%, \beta$ of about $11.5 \%$, and a pitch of about $2 \mu \mathrm{m}$ : crosshatch lines joining the pits. (c) Ge content of about $30.6 \%, \beta$ of about $68.1 \%$, and a pitch of about $2.5 \mu$ m: a conventionally cross-hatched surface indicating that the dislocation distribution is no longer controlled by the pattern.

relaxation. ${ }^{15,16}$ The presence of a dislocation network usually results in a characteristic surface morphology, a perpendicular step lines pattern known as cross hatch that is easily observed by AFM, allowing preliminary experimental characterization. ${ }^{17}$ Figure 1 shows the AFM images of patterns with $700 \mathrm{~nm}$ wide and $300 \mathrm{~nm}$ deep pits with a pit spacing of $2 \mu \mathrm{m}$. For the sample with a Ge content of $12.6 \%$ (Fig. 1(a)), the dislocation density is actually lower than for the other samples (Figs. 1(b) and 1(c)), as due to the lower lattice misfit. The average spacing $D$ of $60^{\circ}$ misfit dislocations required to fully relax a misfit $f$ in a layer with lattice parameter $a(x)$ would be $b \cos \left(60^{\circ}\right) / f$, where the Burger's vector $b$ has length $a(x) / \sqrt{ } 2$, so for an incomplete degree of relaxation $\beta$ in a Sirich $\mathrm{Si}_{1-x} \mathrm{Ge}_{x}$ film on $\mathrm{Si}, D$ is expected to be $\approx 4.8 \mathrm{~nm} /(\beta x)$. However, only a few surface step lines are visible, connecting the corners of adjacent pits. Increasing the Ge content up to $20.4 \%$ leads to a higher dislocations density, as is shown in Fig. 1(b). The lines connecting the corners of the pits are attributed to the piling-up of $60^{\circ}$ dislocations, which propagate along pit rows, ${ }^{18,19}$ according to Ref. 8. The preferential nucleation of dislocation loops at the edges of the pits results into their confinement in stripes and to square areas free of dislocations. On further increasing the Ge content $(x=30.6 \%)$, the dislocation density increases again, making their control ineffective (Fig. 1(c)). The related increase in $\beta$ also gives rise to a larger value of the surface roughness. In particular, the mean square roughness (rms) is about $0.5 \mathrm{~nm}$ for $x=12.6 \%$ (Fig. 1(a)), $1 \mathrm{~nm}$ for $20.4 \%$ (Fig. 1(b)), and $1.5 \mathrm{~nm}$ for $30.6 \%$ (Fig. 1(c)).

In order to stay on the safe side, we set the Ge content to $16 \%$, and the effect of the pit spacing was investigated: structures with different pitches (from 1 up to $10 \mu \mathrm{m}$ ) were realized to study the effectiveness of dislocation gettering by pits. Indeed, a dislocation glide velocity of about $10 \mu \mathrm{m} / \mathrm{s}$ can be estimated, according to the strain in the SiGe film and the growth temperature. ${ }^{13,20}$ Considering that the full SiGe film is deposited in $30 \mathrm{~s}$ there is plenty of time for dislocation loop extension across the pit distance. Figure 2 shows the AFM images of patterns with different pitches $(6,7$, and $8 \mu \mathrm{m})$, and demonstrates that dislocation localization in stripes is maintained even for a pitch of $8 \mu \mathrm{m}$.

The growth temperature is extremely important, since higher temperatures activate both the nucleation and the glide of dislocations, ${ }^{12}$ also increasing the mobility of adatoms, so that we expect a larger degree of relaxation and a larger surface roughness. In particular, the dislocation nucleation rate $J_{\text {nucl }}$ is given by the following empirical formula: ${ }^{13,15,21}$

$$
J_{\text {nucl }}=J_{0} \exp \left(\frac{-E_{T}}{k_{B} T\left|\varepsilon_{\|}\right|}\right),
$$

which results in 4-fold increase between $6500^{\circ} \mathrm{C}$ and $680^{\circ} \mathrm{C}$, if an effective activation energy $E_{T}$ of $26 \mathrm{meV}$ is used. However, it can also be seen that $J_{\text {nuсl }}$ is extremely sensitive to the Ge content, via the in-plane strain $\varepsilon_{\|}$. Samples having the same Ge content $\sim 20 \%$ and SiGe thickness $\sim 190 \mathrm{~nm}$ were grown at different temperatures $\left(550-680^{\circ} \mathrm{C}\right)$. We observe that the dislocation localization induced by the pattern is no longer effective when the growth temperature is $680^{\circ} \mathrm{C}$. By increasing the growth temperature, also increases the surface roughness: from $0.5 \mathrm{~nm}$ to $1.2 \mathrm{~nm}$. In summary, the as-grown samples indicate that the optimum growth temperature to have metastable coherent areas up to $8 \mu \mathrm{m}$ inside is $650^{\circ} \mathrm{C}$, as shown in Fig. 1(b). By considering device possible applications on top or overgrowth with larger Ge contents (e.g., graded layers), it is important to understand whether the metastable areas free of dislocations can be
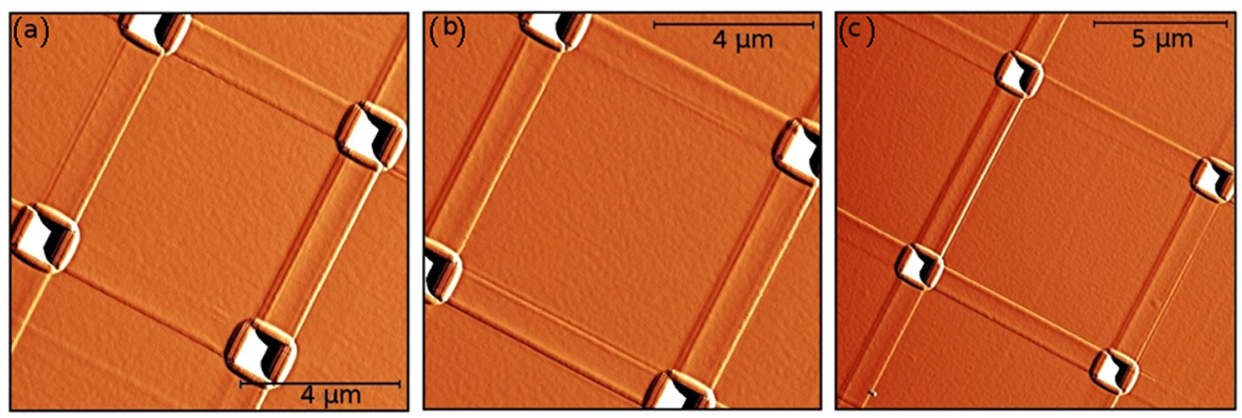

FIG. 2. AFM tapping-amplitude images of the sample with a Ge content of about $16 \%$ and a plastic relaxation of about $2.55 \%$. Patterns with a pitch of: (a) $6 \mu \mathrm{m}$, (b) $7 \mu \mathrm{m}$, and (c) $8 \mu \mathrm{m}$. 
maintained even after high temperature treatments. Pit patterned samples (Ge content $\sim 10 \%-20 \%$ ) were therefore exposed to two different rapid-thermal annealing treatments. In the first case, the temperature was increased to $800^{\circ} \mathrm{C}$ and then immediately cooled down to room temperature; in the second case, the sample was left for $5 \mathrm{~min}$ at $800^{\circ} \mathrm{C}$. AFM, $\mu$ Raman, and X-ray diffraction analyses have been carried out after both annealing treatments: in both cases, the signature of dislocation pile-up in coherent areas did not appear, and the film average degree of relaxation did not significantly change, indicating that such configuration is quite stable, for kinetic reasons.

Since direct dislocation imaging by transmission electron microscopy is not statistically relevant, diffraction experiments on the sample with a Ge content of about $20.4 \%$ and a plastic relaxation of about $11.5 \%$ (Fig. 1(b)) were performed using a nano-focused X-ray beam at the ID01 beamline of the European Synchrotron Radiation Facility in Grenoble. Using quicK continuous Mapping (K-Map), ${ }^{8,11}$ diffraction intensity maps in real space were obtained, and compared with the AFM data and the $\mu$ Raman. The K-Map method allows two-dimensional diffraction intensity maps to be obtained, as shown in Fig. 3. Scanning was carried out both in the symmetric (004) and asymmetric (113) directions, the latter in grazing exit in order to minimize the beam footprint on the sample. ${ }^{8}$ The nano-diffraction results show that the SiGe alloy in the patterned area is more relaxed and more defective along the direction connecting the pits than the SiGe alloy grown between the pits. Figure 3(b) reveals that $q_{x}$ does not show strong variations in the region on the right of the patterned area, indicating that there are no strain
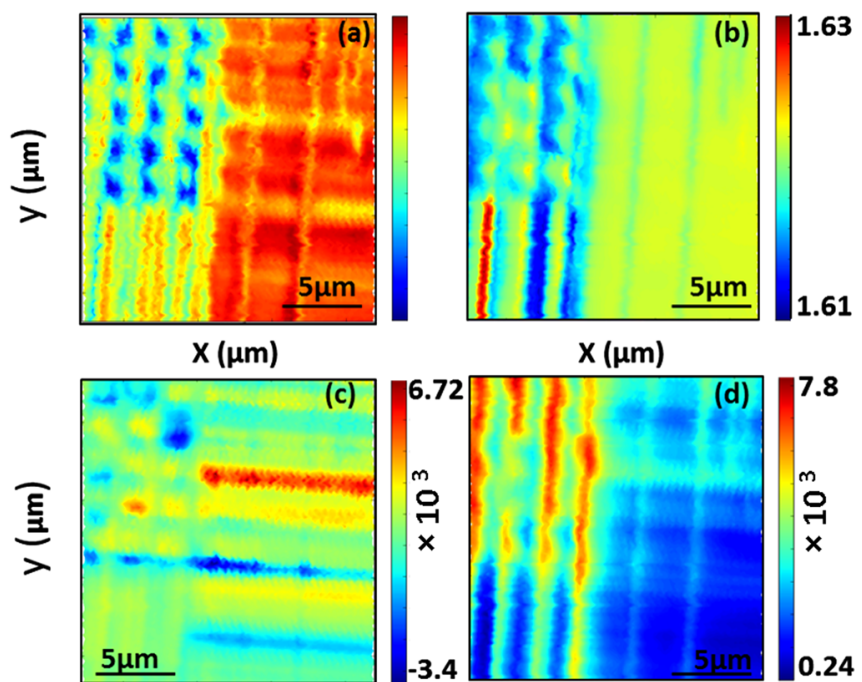

$X(\mu \mathrm{m})$ (c)

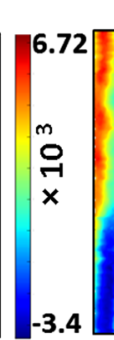

$X(\mu \mathrm{m})$

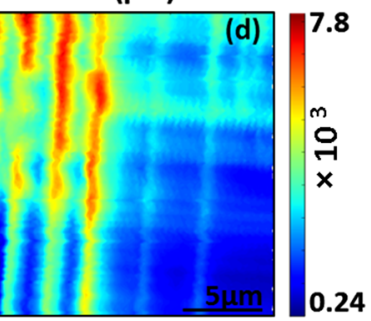

$X(\mu \mathrm{m})$
FIG. 3. (a) X-ray intensity map taken at the corner of the pit-patterned area. The incidence angle corresponds to the SiGe(113) Bragg condition in the unpatterned film. The color scale indicates the logarithm of the intensity (in arbitrary units), increasing from blue to red. Pits are visible as blue areas, corresponding to less intensity at the Bragg angle of the SiGe film. The measurement is sensitive to strain and tilt variations in [110] (defined as the $\mathrm{x}$ direction) from defects running along $[\overline{1} 10]$ (the y direction), and sensitive to tilting towards $[\overline{1} 10]$ (y) from defects running along [110] (x). The position of the SiGe peak in reciprocal space is indicated in panels (b), (c), and (d), which show the $\mathrm{q}_{\mathrm{x}}, \mathrm{q}_{\mathrm{y}}$, and $\mathrm{q}_{\mathrm{z}}$ components of the scattering vector, respectively (with $\mathrm{z}$ corresponding to the [001] direction). variations along $x$ (the [110] direction). However, strong variations can be seen in the region below the pattern, where defects run along $y$ (the $[\overline{1} 10]$ direction). These variations are due to both strain and tilt by dislocations, but the area in Fig. 3(c) to the right of the pattern shows only the tilt arising from defects running along $x . q_{z}$ is sensitive to strain but relatively insensitive to tilt, so the red regions in Fig. 3(d) correspond to relaxation of strain as compared to the blue, strained, regions. It can therefore be seen that the relaxation follows the lines of pits. However, it is difficult to extract the strain state of the regions between pits due to the limit of the $\mathrm{x}$-ray spot size.

To assess it from a quantitative point of view, $\mu$ Raman analyses were carried out on similar samples. $\mu$ Raman spectroscopy, indeed, can monitor local strain variations on a microscopic scale, since the phonon frequency depends on the strain state. ${ }^{22}$ Here, $\mu$ Raman has been used to determine the deformation state of the material and quantify the pattern of partial relaxation induced by the confined dislocations. Using excitation energy of $2.3 \mathrm{eV}$, it is possible to illuminate simultaneously both the epilayer and the substrate. Considering the sample with a Ge content of about $20.4 \%$, plastic relaxation of about $11.5 \%$ and a pitch of about $3 \mu \mathrm{m}$, the map of the Si-Si phonon wavenumber variation in the epilayer can be found in Fig. 4(a): since the penetration depth of the excitation wavelength in SiGe is much larger than the epilayer thickness, the resulting information is an average of the whole epilayer. The large brighter areas indicate a compressive strain, which is closer to the strain of a coherent epilayer, while the darker stripes indicate relaxation, due to the piling-up of confined dislocations. The region of the pit is actually more relaxed, because the slanted free facets allow the epilayer to relax elastically. ${ }^{23}$ Figure 4(b) shows the corresponding map of the Si-Si phonon wavenumber from the Si substrate. In this case, the penetration depth is about $1 \mu \mathrm{m}$, and therefore the results are averaged over a spatial region, which is much larger than the case of the epilayer. The regions of substrate away from the dislocation patterns are completely relaxed, while the regions under the dislocations are under slightly compressive strain, because of the negative lobes of the dislocation strain field heading towards the substrate. ${ }^{24}$ The quantification of the strain from the Raman shift requires some assumptions about the strain
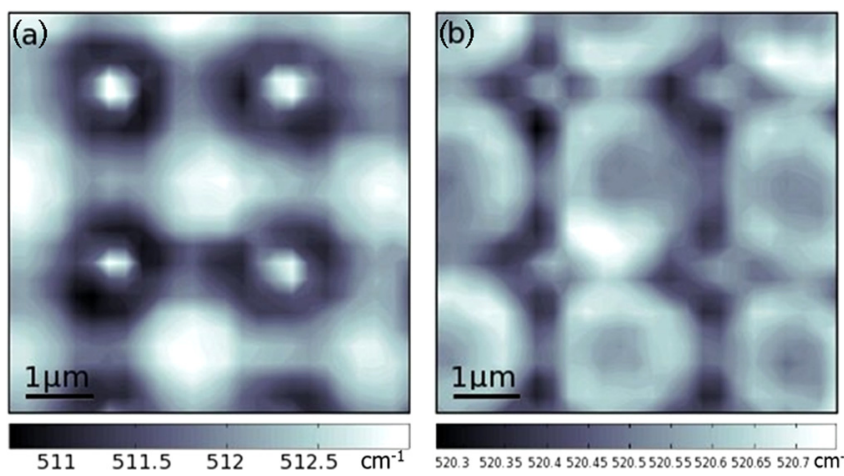

$520.3520 .35520 .4520 .45520 .5520 .55520 .6520 .65520 .7 \mathrm{~cm}^{-1}$

FIG. 4. Raman spectroscopy map of a pit-patterned area with a pitch of $2.5 \mu \mathrm{m}$. (a) Si-Si peak of the SiGe film. This mode in fully strained $\mathrm{SiGe}$ at this composition of $x=20.4 \%$ is centered at $513.3 \mathrm{~cm}^{-1}$. (b) Si-Si peak of the $\mathrm{Si}$ substrate. This mode in unstrained $\mathrm{Si}$ is centered at $520.7 \mathrm{~cm}^{-1}$. 
tensor. If we consider similar deformation potentials for $\mathrm{Si}$ and $\mathrm{SiGe},{ }^{25,26}$ a variation of $+1 \mathrm{~cm}^{-1}$ corresponds to a change of uniaxial strain of about $-0.26 \%$ or a change of biaxial strain of $-0.13 \%$. Simple symmetry arguments suggest that due to the localized dislocation distribution, the strain should be almost uniaxial close in the stripes along the pit lines, decreasing to a very low biaxial value close to the center of the squares with pits at the corners.

In conclusion, we demonstrated that the pit spacing, the growth temperature, the SiGe thickness, and the Ge content can be tailored to get full dislocation localization along the pit lines, so that the square areas delimited by such dislocated stripes are fully coherent. Rapid thermal treatments show that such configuration is kinetically stable against thermal budgets, so that device fabrication on stressed SiGe areas, overcritical in thickness can be attempted, or overgrowth with graded SiGe layers, in order to study spatial the distribution of the additional threading arms. The use of a graded layer, in which misfits are also distributed vertically rather than all being at the $\mathrm{SiGe} / \mathrm{Si}$ interface, may allow higher plastic relaxation while maintaining a wide horizontal spacing between misfits. We think these further issues to be interesting both for applications and for fundamental understanding of dislocation nucleation in graded layers.

We acknowledge the Cariplo Foundation (within the Grant No. DefCon4 2011-0331) for partial financial support.

${ }^{1}$ M. Ieong, B. Doris, J. Kedzierski, K. Rim, and M. Yang, Science 306, 2057 (2004).

${ }^{2}$ S. C. Jain, S. Decoutere, M. Willander, and H. E. Maes, Semicond. Sci. Technol. 16, R51 (2001).

${ }^{3}$ GeSi Strained Layers and their Applications, edited by A. M. Stoneham and S. C. Jain (CRC Press; Reprint edition, 1995).

${ }^{4}$ S. E. Thompson, G. Sun, Y. S. Choi, and T. Nishida, IEEE Trans. Electron Devices 53, 1010 (2006).

${ }^{5}$ R. People and J. C. Bean, Appl. Phys. Lett. 47, 322 (1985).

${ }^{6}$ J. G. Fiorenza, G. Braithwaite, C. W. Leitz, M. T. Currie, J. Yap, F. Singaporewala, V. K. Yang, T. A. Langdo, J. Carlin, M. Somerville, A.
Lochtefeld, H. Badawi, and M. T. Bulsara, Semicond. Sci. Technol. 19, L4 (2004).

${ }^{7}$ M. Grydlik, F. Boioli, H. Groiss, R. Gatti, M. Brehm, F. Montalenti, B. Devincre, F. Schäffler, and L. Miglio, Appl. Phys. Lett. 101, 013119 (2012).

${ }^{8}$ V. Mondiali, M. Bollani, S. Cecchi, M. Richard, T. Schülli, G. Chahine, and D. Chrastina, Appl. Phys. Lett. 104, 021918 (2014).

${ }^{9}$ J. Goldberger, A. I. Hochbaum, R. Fan, and P. Yang, Nano Lett. 6, 973 (2006).

${ }^{10}$ J. Michel, J. Liu, and L. C. Kimerling, Nat. Photonics 4, 527 (2010).

${ }^{11}$ G. A. Chahine, M.-I. Richard, R. A. Homs-Regojo, T. N. Tran-Caliste, D. Carbone, V. L. R. Jaques, R. Grifone, P. Boesecke, J. Katzer, I. Costina, H. Djazouli, T. Schroeder, and T. U. Schülli, J. Appl. Cryst. 47, 762 (2014).

${ }^{12}$ M. Bollani, S. Bietti, C. Frigeri, D. Chrastina, K. Reyes, P. Smereka, J. M. Millunchick, G. M. Vanacore, M. Burghammer, A. Tagliaferri, and S. Sanguinetti, Nanotechnology 25, 205301 (2014).

${ }^{13}$ J. Frigerio, M. Lodari, D. Chrastina, V. Mondiali, G. Isella, and M. Bollani, J. Appl. Phys. 116(11), 113507 (2014).

${ }^{14}$ A. Fischer, H. Kühne, B. Roos, and H. Richter, Semicond. Sci. Technol. 9, 2195 (1994).

${ }^{15}$ T. J. Gosling, S. C. Jain, and A. H. Harker, Phys. Status Solidi A 146, 713 (1994).

${ }^{16}$ M. Bollani, D. Chrastina, M. Fiocco, V. Mondiali, J. Frigerio, L. Gagliano, and E. Bonera, J. Appl. Phys. 112, 094318 (2012).

${ }^{17}$ M. Kummer, B. Vögeli, T. Meyer, and H. von Känel, Phys. Rev. Lett. 84, 107 (2000).

${ }^{18}$ M. A. Lutz, R. M. Feenstra, F. K. LeGoues, P. M. Mooney, and J. O. Chu, Appl. Phys. Lett. 66, 724 (1995).

${ }^{19}$ A. Fischer, H. Kühne, M. Eichler, F. Holländer, and H. Richter, Phys. Rev. B 54, 8761 (1996).

${ }^{20}$ C. G. Tuppen and C. J. Gibbings, J. Appl. Phys. 68(4), 1526-1534 (1990).

${ }^{21}$ G. G. Fischer and P. Zaumseil, Phys. Status Solidi A 164(2), 767-778 (1997).

${ }^{22}$ F. Pezzoli, E. Bonera, E. Grilli, M. Guzzi, S. Sanguinetti, D. Chrastina, G. Isella, H. von Känel, E. Wintersberger, J. Stangl, and G. Bauer, Mater. Sci. Semicond. Process. 11, 279 (2008).

${ }^{23}$ F. Boioli, R. Gatti, M. Grydlik, M. Brehm, F. Montalenti, and L. Miglio, Appl. Phys. Lett. 99, 033106 (2011).

${ }^{24}$ E. Bonera, R. Gatti, G. Isella, G. Norga, A. Picco, E. Grilli, M. Guzzi, M. Texier, and B. Pichaud, Appl. Phys. Lett. 103, 053104 (2013).

${ }^{25}$ F. Pezzoli, E. Bonera, E. Grilli, M. Guzzi, S. Sanguinetti, D. Chrastina, G. Isella, H. von Känel, E. Wintersberger, J. Stangl, and G. Bauer, J. Appl. Phys. 103, 093521 (2008).

${ }^{26}$ E. Bonera, M. Bollani, D. Chrastina, F. Pezzoli, A. Picco, O. G. Schmidt, and D. Terziotti, J. Appl. Phys. 113(16), 164308 (2013). 